\title{
3, 4-Dihydroxyphenylethanol attenuates cadmium-induced oxidative stress, inflammation and apoptosis in rat heart
}

\author{
Yan Huang ${ }^{1 *}$, Hongsheng Gang ${ }^{2}$, Bitao Liang ${ }^{3}$, Ming Li $^{1}$ \\ ${ }^{1}$ Department of Emergency, ${ }^{2}$ Department of Vasculocardiology, Wuhan Fourth Hospital (Puai Hospital),Tongji Medical College, \\ Huazhong University of Science and Technology, ${ }^{3}$ Changjiang Polytechnic, Wuhan 430073, Hubei, China
}

*For correspondence: Email: holstermarobit@yahoo.com; Tel/Fax: 0086-027-83914556

\begin{abstract}
Purpose: To investigate the therapeutic effect of 3, 4-dihydroxyphenylethanol (DOPET) on cadmium (Cd) induced cardiotoxicity in murine model.

Methods: Four groups of rats were used in this study $(n=6)$. The rats were treated with DOPET and Cd for 28 days. Biochemical parameters were determined in plasma and heart tissue homogenates.

Results: Cadmium (Cd) significantly increased lipid peroxidation and protein carbonylation. However, DOPET treatment significantly attenuated $\mathrm{Cd}$-induced oxidative stress. Cd intoxication significantly increased cardiac markers \{creatine kinase, lactate dehydrogenase (LDH) and cardiac troponin-l\} levels in plasma, and reduced the levels of antioxidants such as superoxide dismutase (SOD), catalase (CAT), glutathione peroxidase Gpx, glutathione (GSH) and malndialdehyde (MDA) in heart tissue. These Cdinduced changes in cardiac markers and antioxidants were reversed by DOPET treatment. Cd treatment led to upregulation of protein expressions of pro-inflammatory cytokines (TNF- $\alpha$ and II-6). However, DOPET supplementation brought about a decrease in the protein expressions of these cytokines. Western blot analysis revealed that Cd induced apoptosis in cardiac tissue, as was evident from alterations in protein expressions of the apoptotic inducers, Bax and cleaved caspase-3, and the anti-apoptotic factor Bcl-2. However, DOPET treatment effectively reversed $\mathrm{Cd}$-induced apoptosis.

Conclusion: These results indicate that DOPET exerts cardio-protective effect against Cd-induced toxicity via antioxidant, anti-inflammatory and anti-apoptotic mechanisms.
\end{abstract}

Keywords: Cadmium, Cardiotoxicity, DOPET, Antioxidant, Inflammation, Apoptosis

This is an Open Access article that uses a fund-ing model which does not charge readers or their institutions for access and distributed under the terms of the Creative Commons Attribution License (http://creativecommons.org/licenses/by/4.0) and the Budapest Open Access Initiative (http://www.budapestopenaccessinitiative.org/read), which permit unrestricted use, distribution, and reproduction in any medium, provided the original work is properly credited.

Tropical Journal of Pharmaceutical Research is indexed by Science Citation Index (SciSearch), Scopus, International Pharmaceutical Abstract, Chemical Abstracts, Embase, Index Copernicus, EBSCO, African Index Medicus, JournalSeek, Journal Citation Reports/Science Edition, Directory of Open Access Journals (DOAJ), African Journal Online, Bioline International, Open-J-Gate and Pharmacy Abstracts

\section{INTRODUCTION}

Cadmium $(\mathrm{Cd})$ is a toxic transition heavy metal which acts as a pro-inflammatory agent and a potent carcinogen. As an environmental and occupational toxicant, humans are exposed to $\mathrm{Cd}$ in various staple food items like rice, wheat, peanuts, and grains through soil contamination from various chemical industries [1]. Furthermore, tobacco smoke contributes to human exposure to $\mathrm{Cd}$. Cadmium is accumulated in its soluble form, cadmium chloride $\left(\mathrm{CdCl}_{2}\right)$. It exerts noxious effects in various tissues and organs such as liver, kidney, brain and heart. 
Globally, cardiovascular diseases (CVD) remain the major cause of mortality, despite the advancement in treatment approaches [2]. Environmental changes, high fat diet and lack of exercise remain cardinal factors in the pathogenesis of CVD. Studies have shown that environmental pollution is a key factor in CVD [3]. Increasing evidence have highlighted the relationship between $\mathrm{Cd}$ exposure and increased risk of stroke, a CVD [5]. Preclinical studies have shown that $\mathrm{Cd}$ insult can lead to cardiotoxicity [5]. Cadmium (Cd) exerts cardiotoxicity at concentrations as low as $0.1 \mathrm{mM}$ [6]. Cadmiuminduced cardiotoxicity is mediated through generation of reactive oxygen species (ROS) which in turn decrease the levels of antioxidants [6]. The ROS induce membrane lipid peroxidation, and damage proteins, DNA and other vital biomolecules. Furthermore, Cdinduced ROS increases oxidative stress in the myocardial mitochondria which lead to loss of membrane integrity, activation of caspases and apoptosis of myocytes [7].

The use of plants for the treatment of CVD has continued to attract increasing attention due to their proven efficacy and lack of adverse effects. Polyphenols have shown promising effects in attenuating oxidative stress-mediated cardiac injury. In addition, antioxidants from natural sources have been shown to protect various organs and tissues from $\mathrm{Cd}$-induced oxidative cellular damage [8]. Hydroxytyrosol (3, 4dihydroxyphenylethanol, DOPET) is a potent polyphenol present in the olive oil [9]. Previous studies have demonstrated the protective effect of DOPET against oxidative stress-mediated cellular death [10]. Moreover, it has been reported that DOPET exerts protective effect on heavy metal-induced neurotoxicity [11]. Earlier reports showed the protective influence of the antioxidant potential of DOPET against $\mathrm{Cd}$ induced oxidative insult [12]. Recently, it was shown that DOPET exerted cardio-protective effect against ischemic/reperfusion and isoproterenol-induced myocardial infarction through its free radical scavenging potential [13]. The current study was undertaken to investigate the protective effect of DOPET against $\mathrm{Cd}$ provoked oxidative cardiotoxicity by assessing various biochemical parameters.

\section{EXPERIMENTAL}

\section{Drugs and chemicals}

Hydroxytyrosol (DOPET) and Cd were procured from Sigma Chemicals, USA. The other reagents used in the study were analytical grade, and were purchased from Merck, USA.

\section{Animals}

Male Sprague Dawley Wistar rats aged 6 weeks and weighing about $180-200 \mathrm{~g}$ were utilized for the study. The animals were obtained from Tongji Medical College, Huazhong University of Science and Technology, Wuhan, Hubei, China. The rats were housed in polypropylene cages under standard laboratory conditions at a temperature of $25^{\circ} \mathrm{C}$ and alternating $12 \mathrm{~h}$ light/12 $\mathrm{h}$ dark cycle. The animals were allowed free access to water and pelleted rat feed. All animal studies were carried out as per the protocol outlined in Care and Use of Laboratory Animals, NIH. The study received approval from the Ethical Committee for Animal Care and Use, Huazhong University of Science and Technology, Wuhan, Hubei, China (Approval no: TK2016321).

\section{Experimental design}

Three groups of rats were used (6 rats per group). Group 1 rats were treated with normal saline, and served as control, while group 2 rats received cadmium chloride $\left(\mathrm{CdCl}_{2}\right)$ at a dose of 5 $\mathrm{mg} / \mathrm{kg}$ body weight (b.wt.) in normal saline, via the intra-gastric route. Group 3 rats were given DOPET (10 mg/kg b.wt) orally, while in group 4, the rats were pretreated with DOPET $(10 \mathrm{mg} / \mathrm{kg}$; b.wt) $1 \mathrm{~h}$ before administration of $\mathrm{CdCl}_{2}(5 \mathrm{mg} / \mathrm{kg}$ b.wt). All treatments were given daily for 28 days.

After the final doses of DOPET and Cd, the rats were subjected to overnight fast. Thereafter, they were anaesthetized using phenobarbital sodium at the dose of $35 \mathrm{mg} / \mathrm{kg}$, i.p. and sacrificed through cervical dislocation. Blood was withdrawn from the jugular vein in heparinized tubes and the plasma was separated for use in the measurement of cardiac marker enzymes. The heart tissue was excised, cleaned from adherent tissues, washed in ice-cold saline and blotted. Then, a $10 \%(w / v)$ tissue homogenate was prepared by homogenizing $100 \mathrm{mg}$ of cardiac tissue in chilled Tris- $\mathrm{HCl}$ buffer. The homogenate was used for the assays of various biochemical markers of Cd-induced cardiac damage.

\section{Assay of cardiac markers}

Creatinine kinase-MB (CK-MB) and lactate dehydrogenase (LDH) levels were measured in plasma using standard kits from Nanjing Jiancheng Bio-Engineering Institute, Nanjing, China. Furthermore, plasma cardiac troponin (cTn) cTn-I was estimated with ELISA kits (Biocheck ELISA kit, USA) according to manufacturer's protocol. 
Evaluation of lipid peroxidation and protein carbonyl content

The lipid peroxidation (LPO) index, malondialdehyde (MDA) was estimated spectrophotometrically at $532 \mathrm{~nm}$ using the method of Ohkawa et al [14]. The protein carbonyl content was quantified using the method of Levine et al [15].

\section{Assay of antioxidants}

The cardiac level of antioxidants CAT, SOD, GPx and GSH were measured using assay kits (Nanjing Jiancheng Bio-Engineering Institute, Nanjing, China) in line with the instructions on the kit manuals.

\section{Determination of plasma pro-inflammatory cytokines}

Plasma levels of pro-inflammatory cytokines TNF- $\alpha$ and IL- 6 were estimated using ELISA kit in accordance to the instruction provided in the protocol by the manufacturer (Sigma Aldrich, USA).

\section{Western blot analysis of pro-inflammatory cytokines and apoptosis markers}

Heart tissue lysate samples were collected using RIPA lysis buffer kit (Applygen Technologies Inc., China). Next, $20 \mu \mathrm{g}$ of protein was subjected to $10 \%$ sodium dodecyl sulfate polyacrylamide gel electrophoresis (SDS-PAGE). After immunoblotting, the separated proteins were transferred to a nitrocellulose membrane, washed, and incubated with anti-IL-6, anti-TNF$\alpha$, anti-Bax, anti-cleaved caspase- 3 and anti-Bcl2 antibodies. Following incubation with primary antibodies, the nitrocellulose membranes were incubated with peroxidase-conjugated goat antimouse IgG secondary antibody (Santa Cruz, USA). Visualization of proteins was done using ECL detection kit (Applygen Technologies Inc,
China) and the imaging and analysis of the protein bands were done with Gel Doc XR system (Bio-Rad, USA).

\section{Statistical analysis}

The data are presented as mean \pm standard error of the mean (SEM). One-way analysis of variance, followed Tukey's comparison test using SPSS version 18.0 was performed for comparison amongst the groups. Values of $p<$ 0.05 were assumed statistically significant.

\section{RESULTS}

\section{Effect of DOPET administration on cardiac markers}

The effect of DOPET treatment on levels of cardiac markers in the Cd-toxified rats is shown in Table 1. There were significant elevations in the plasma concentrations of cardiac markers CK-MB, LDH and cardiac troponin I (cTn-I) $(p<$ $0.05)$. Treatment with DOPET effectively reduced the plasma levels of the cardiac markers to normal.

\section{Effect of DOPET on cardiac lipid peroxidation and protein oxidation}

As shown in Figure $1 \mathrm{~A}$, the Cd-intoxicated rats showed significantly increased lipid peroxidation as evident from increased MDA content, relative to the control group. However, DOPET administration reversed the Cd-induced increases in MDA to normal levels $(p<0.05)$. The cardiac tissue level of protein carbonyl, an end product of protein oxidation was significantly increased in the Cd-intoxicated group, when compared to the control group. However, DOPET treatment reversed the Cd-induced protein oxidation and restored the cardiac tissue protein carbonyl to normal level (Figure $1 \mathrm{~B}$ ).

Table 1: Effect of DOPET and Cd on plasma cardiac markers

\begin{tabular}{|c|c|c|c|}
\hline Group & LDH (IU/L) & CK-MB (IU/L) & cTnl $(\mu \mathrm{g} / \mathrm{ml})$ \\
\hline Control & $145.34 \pm 5.43$ & $103.76 \pm 4.32$ & $1.23 \pm 0.23$ \\
\hline $\mathrm{Cd}$ & $567.98 \pm 10.76^{a^{\circ}}$ & $456.32 \pm 9.76^{a^{a}}$ & $3.43 \pm 0.56^{a^{a}}$ \\
\hline DOPET & $148.76 \pm 4.65$ & $105.87 \pm 4.32$ & $1.25 \pm 0.26$ \\
\hline DOPET + Cd & $176.65 \pm 4.67^{\mathrm{b}^{x}}$ & $112.43 \pm 6.78^{b^{x}}$ & $1.41 \pm 0.35^{\mathrm{b}^{\pi}}$ \\
\hline
\end{tabular}

Values are shown as mean \pm SEM $(n=6)$. Analyses were done by one-way analysis of variance (ANOVA) followed by Tukey's post-hoc comparison test. The comparison were made between Cd group and control (a); and between Cd group and DOPET+ Cd group (b); ${ }^{*} p<0.05$ 

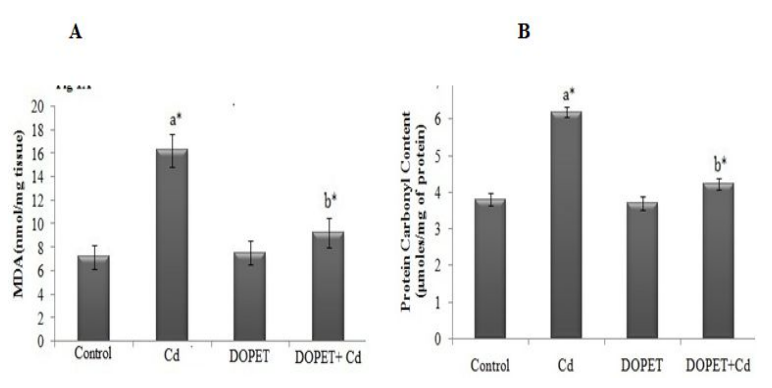

Figure 1: Effect of DOPET on lipid peroxidation and protein carbonyl level in heart tissue. Cd induced oxidative stress and caused marked increase in MDA level and protein carbonyl content in cardiac tissue. DOPET treatment effectively decreased the lipid peroxidation and protein carbonyl levels. A: MDA; B: Protein carbonyl content. Data are shown as mean \pm SEM $(n=6)$. Comparison was made between Cd group and control (a), and between b-DOPET+ Cd group and Cd group (b); ${ }^{*} p<0.05$

\section{Effect of DOPET on antioxidant status in Cd- induced cardiac oxidative stress}

There were significant decreases in cardiac tissue levels of SOD, CAT, GPx and GSH in the Cd-intoxicated rats, when compared to the control group $(p<0.05)$. However, DOPET treatment significantly reversed the Cd-induced decreases in these antioxidants, relative to the Cd group $(p<0.05)$. The results are shown in Table 2.

\section{Effect of DOPET on pro-inflammatory cytokine levels in Cd-induced cardiotoxicity}

The plasma levels of pro-inflammatory cytokines (TNF- $\alpha$ and II-6) were significantly elevated in Cd group, when compared to the control group. The administration of DOPET significantly reduced the Cd-induced increases in pro-inflammatory cytokine levels to normal (Figure 2).

\section{Effect of DOPET on protein expressions of TNF- $\alpha$ and II-6 in cardiac tissue}

The protein expressions of TNF- $\alpha$ and II- 6 were significantly upregulated in Cd-toxified rats, when compared to the control rats (Figure $3 \mathrm{~A}$ ).
Furthermore, the relative protein levels of TNF-a, II-6 and IL1- $\beta$ were significantly increased in the $\mathrm{Cd}$ group, relative to the control group. However, administration of DOPET significantly decreased the protein expressions of pro-inflammatory cytokines, when compared to the $\mathrm{Cd}$ group ( $p<$ 0.05; Figure $3 \mathrm{~B}$ ).
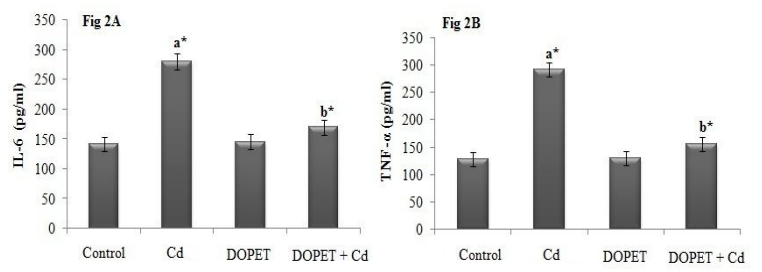

Figure 2: Effect of DOPET on plasma levels of proinflammatory cytokines in Cd-induced cardiotoxicity. Cd-treated rats showed elevated levels of IL-6 (A) and TNF- $\alpha$ (B). DOPET-treated rats showed reduced levels of pro-inflammatory cytokines. The values are shown as mean \pm SEM $(n=6)$. Analysis was done by one-way analysis of variance (ANOVA) followed by Tukey's post-hoc comparison test. Comparison was made between $\mathrm{Cd}$ group and control (a), and between b-DOPET+ Cd group and Cd group (b); ${ }^{*} p<0.05$ )
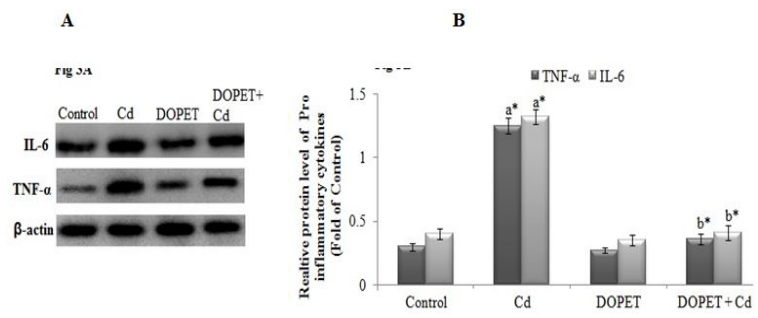

Figure 3: Effect of DOPET on protein expressions of pro-inflammatory cytokines in Cd-induced cardiotoxicity. A: Cd group showing up-regulated expressions of pro-inflammatory cytokines; DOPET treatment effectively down-regulated the protein expressions. B: Significantly reduced relative protein expressions of pro-inflammatory cytokines in DOPETtreated, Cd-exposed rats $(p<0.05)$. Values are expressed as mean \pm SEM $(n=6)$. Analyses were done using one way analysis of variance (ANOVA) followed by Tukey's post-hoc comparison test. Comparison was made between $\mathrm{Cd}$ group and control; and between b-DOPET+Cd group and Cd group; ${ }^{*} p<$ 0.05

Table 2: Effect of DOPET and Cd on antioxidant levels in heart homogenate

\begin{tabular}{|c|c|c|c|c|}
\hline Group & SOD & CAT & GPx & GSH \\
\hline Control & $10.34 \pm 0.87$ & $7.54 \pm 0.45$ & $20.54 \pm 1.24$ & $14.27 \pm 1.26$ \\
\hline $\mathrm{Cd}$ & $5.98 \pm 0.566^{a^{\pi}}$ & $3.12 \pm 0.23^{\mathrm{a}^{x}}$ & $12.56 \pm 1.12^{a^{x}}$ & $7.15 \pm 0.82^{a^{\pi}}$ \\
\hline DOPET & $11.54 \pm 0.76$ & $7.28 \pm 0.38$ & $19.86 \pm 1.54$ & $13.58 \pm 1.12$ \\
\hline DOPET+ Cd & $8.87 \pm 0.62^{b^{\wedge}}$ & $6.98 \pm 0.36^{\mathrm{b}^{-}}$ & $18.34 \pm 1.28^{\mathrm{b}^{\wedge}}$ & $12.54 \pm 1.42^{b^{n}}$ \\
\hline
\end{tabular}

Values are shown as mean \pm SEM $(n=6)$. Analyses were performed using ANOVA followed by Tukey's post-hoc comparison test. Comparison was made between $\mathrm{Cd}$ group and control (a), and between b-DOPET+ Cd group and Cd group; ${ }^{*} p<0.05$. Units: SOD: U/mg protein; CAT: $\mu$ moles $/ \mathrm{H}_{2} \mathrm{O}_{2} / \mathrm{min} / \mathrm{mg}$ protein; GPx: $\mu$ moles NADPH oxidized /min/mg protein; GSH: nmol/mg protein 


\section{Effect of DOPET on apoptotic protein expressions in cardiac tissue}

The protein expressions of the apoptotic markers Bax and cleaved caspase-3 were upregulated, while Bcl-2 was downregulated in Cd-toxified rats, when compared to the control group (Figure 4 A). However, DOPET treatment significantly downregulated the protein expressions of Bax and cleaved caspase-3, and upregulated the protein expression of $\mathrm{Bcl}-2$, when compared to the Cd group (Figure $4 \mathrm{~B}$ ).

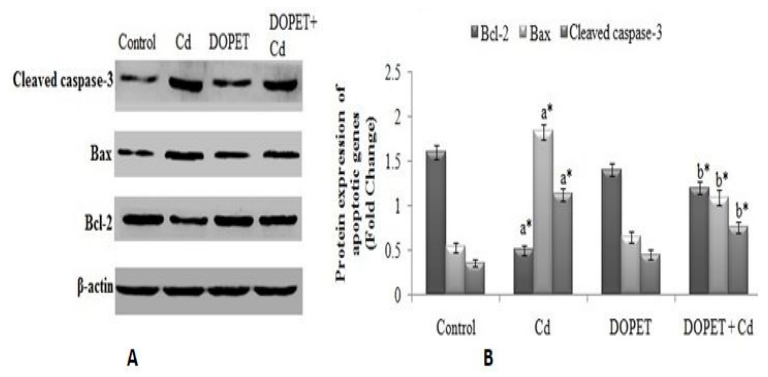

Figure 4: Effect of DOPET on the protein expressions of apoptotic markers in $\mathrm{Cd}$-induced cardiotoxicity. A: $\mathrm{Cd}$ treatment led to upregulation of the expressions of Bax and cleaved caspase-3, whilst $\mathrm{Bcl}-2$ protein expression was down-regulated. DOPET treatment effectively reversed the altered protein expressions. B: Relative protein expressions of apoptotic genes in $\mathrm{Cd}$ treated rats. The values are shown as mean \pm SEM ( $n$ $=6$ ). Analyses were done with one way analysis of variance (ANOVA) followed by Tukey's post-hoc comparison procedure. Comparison was made between $\mathrm{Cd}$ group and control (a), and between bDOPET+ Cd group and Cd group; ${ }^{*} p<0.05$

\section{DISCUSSION}

Oxidative stress, a condition due to excess production of ROS and loss of cellular antioxidant defense mechanism, is a cardinal factor in an array of cardiovascular diseases. The current study investigated cardio-protective effect of 3, 4-dihydroxyphenylethanol (DOPET) against Cd-provoked oxidative cardiotoxicity. Cadmium (Cd) mediates its toxic effects by generation of ROS which bring about alterations in metabolic pathways involved in energy metabolism, protein synthesis and antioxidant network. Furthermore, Cd affects DNA by altering gene control and repair [21]. It triggers peroxidation of lipids present in cell membrane due to excessive ROS release, thereby decreasing the level cellular antioxidants available for neutralization of free radicals [16].

The clinical diagnosis of the pathological symptoms of $\mathrm{Cd}$ toxicity relies on measurement of cytoplasmic enzymes released into the blood stream during oxidative membrane damage.
Thus, increases in the levels of plasma marker enzymes are indicative of tissue damage and loss of membrane integrity. Creatine kinase (CK$\mathrm{MB}$ ) and LDH are the cardiac marker enzymes used to assess the severity of cardiac damage during oxidative free radical attack. During this process, the cardiac membrane integrity is compromised, leading to leakage of enzymes into the blood stream [17].

Troponin is a cardiac protein located in the thin filaments of striated muscles present in heart tissue. It is composed of three subunits namely troponin T (cTn-T), troponin I (cTn-I) and troponin C. Troponin I (cTn-I) is clinically used to assess the degree of myocardial injury [17]. In the present study, Cd-intoxication caused significant elevation in the level of plasma marker enzymes CM-BK, LDH and cTn-I. This is in agreement with previous reports. However, treatment with DOPET mitigated the Cd-mediated increases in levels of plasma cardiac marker enzymes and cardiac troponin. This effect is due to the free radical-quenching and membrane-stabilizing potential of DOPET [13].

Polyunsaturated fatty acids (PUFA) which are present in all tissues and organs are vulnerable to free radical damage due to their high degree of unsaturation. Oxidative deterioration of polyunsaturated fatty acids is a noxious process involved in cell injury during $\mathrm{Cd}$ exposure. In the present study, Cd-provoked cardiotoxicity was due to increased generation of free radicals in heart tissue, which was evident from the marked increases in the level of MDA, a reliable marker of lipid peroxidation. Cadmium (Cd)-induced lipid peroxidation has been reported in previous studies. Oxidative damage by ROS results in protein oxidation, the end product of which is protein carbonyl. The protein carbonyl content (PCC) is a reliable marker of the extent of protein oxidation. In the present study, administration of DOPET to Cd-intoxicated rats reduced LPO and protein oxidation, and thus protected proteins and lipids from oxidative attack [18].

The non-enzymatic antioxidant, thiol or sulfhydryl $(-\mathrm{SH})$ group is involved in detoxification of $\mathrm{Cd}$ through formation of a complex with $\mathrm{Cd}(\mathrm{Cd}-\mathrm{SH})$. Reduced glutathione (GSH), a thiol compound forms a complex with $\mathrm{Cd}$ through covalent bonding [19]. In the current study, the Cdintoxicated rats had significantly reduced level of $\mathrm{GSH}$, which might be due to utilization of $\mathrm{GSH}$ to quench the free radicals released by $\mathrm{Cd}$ mediated lipid peroxidation, as well as the high affinity binding of $\mathrm{Cd}$ to thiol groups. However, treatment with DOPET reversed the $\mathrm{Cd}$-induced 
decrease in $\mathrm{GSH}$, most likely due to the free radical scavenging potential of DOPET.

Oxidative stress is a pathological condition in which the physiological antioxidant network losses the ability to remove toxic free radicals form the cells. Catalase and SOD and are the mainstay antioxidant enzymes that protect the cells from oxidative damage. Superoxide dismutase (SOD) is involved in the removal of highly reactive superoxide ion, while catalase mediates the detoxification of toxic $\mathrm{H}_{2} \mathrm{O}_{2}$. Thus, SOD and catalase guard the cells/organs from free radical attack. In this study, the cardiac levels of SOD, catalase and GPx were decreased following $\mathrm{Cd}$ intoxication. However, DOPET treatment effectively increased the antioxidant enzyme levels via its antioxidant activity [20]. The Cd-toxified rats showed elevated levels of TNF- $\alpha$ and IL-6. Cadmium activates monocytes and tissue macrophages to secrete pro-inflammatory cytokines [21]. Treatment with DOPET significantly reduced plasma levels of TNF- $\alpha$ and IL-6, thereby mitigating the Cd-induced inflammation [7,22].

Studies have shown $\mathrm{Cd}$-induced toxicity results in apoptotic cells [23] The anti-apoptotic protein $\mathrm{Bcl}-2$, is a key modulator that regulates the intrinsic apoptotic pathway by preventing the release of cytochrome-c from the mitochondria following caspase activation [24]. Caspases are a family of proteases involved in the initiation and activation of apoptotic pathway, with caspase-3 as the key mediator. The proteins $\mathrm{Bcl}-2$ and Bax participate in the caspase activation. Caspase-3 participates in the downstream signalling of the mitochondrial pathway, and its activation results in irreversible apoptosis [25].

In the present research, it was found that the proapoptotic factors Bax and cleaved caspase-3 were upregulated, whereas $\mathrm{BCl}-2$ was decreased in the immunoblot obtained from the $\mathrm{Cd}$ group. However, administration of DOPET attenuated the expressions of these apoptotic proteins. In addition, it was found that treatment with DOPET increased the anti-apoptotic Bcl-2 expression, indicating that amelioration of apoptosis may be a potent therapeutic target in Cd-induced cardiotoxicity.

\section{CONCLUSION}

The findings of this study show that DOPET exerts cardio-protective effect against Cdinduced cardiotoxicity by regulation of cardiac marker enzymes, inhibition of lipid peroxidation, and enhancement of antioxidant status, antiinflammation and anti-apoptotic pathways.

\section{DECLARATIONS}

\section{Acknowledgement}

The authors thank Wuhan Health and Family Planning Commission for funding this project (no. WX16B13).

\section{Conflict of interest}

No competing interest is associated with this work.

\section{Contribution of authors}

We declare that this work was done by the authors named in this article and all liabilities pertaining to claims relating to the content of this article will be borne by the authors. $\mathrm{YH}$ and $\mathrm{HG}$ prepared the protocol and carried out the study. $\mathrm{BL}$ prepared the manuscript and ML performed the statistical analysis

\section{Open Access}

This is an Open Access article that uses a funding model which does not charge readers or their institutions for access and distributed under the terms of the Creative Commons Attribution License (http://creativecommons.org/licenses/by/ 4.0) and the Budapest Open Access Initiative (http://www.budapestopenaccessinitiative.org/rea d), which permit unrestricted use, distribution, and reproduction in any medium, provided the original work is properly credited.

\section{REFERENCES}

1. WHO. Safety evaluation of certain food additives and contaminants. Seventy-third report of the Joint FAO/WHO Expert Committee on Food Additives. 2011. Available from: http://www.Inchem.Org/documents/ jecfa/jecmono/v64je01

2. WHO. Cardiovascular Diseases (CVDs). WHO Media Centre. 2017. Available From: http://www.who.int/nmh/ publications/fact_sheet_cardiovascular_en.pdf

3. Pope CA, Burnett RT, Thurston GD, Thun MJ, Calle EE, Krewski D, Godleski J J. Cardiovascular mortality and long-term exposure to particulate air pollution. Circulation 2004; 109(1): 71-77.

4. Oyinloye BE, Ajiboye BO, Ojo OA, Nwozo SO, Kappo $A P$. Cardio protective and antioxidant influence of aqueous extracts from Sesamum indicum seeds on oxidative stress induced by cadmium in Wistar rats. Pharmacogn Mag 2016; 12 (S2): 170-174.

5. Limaye $D A$, Shaikh ZA. Cytotoxicity of cadmium and characteristics of its transport in Cardiomyocytes. Toxicol Appl Pharmacol 1999; 154(1): 59- 66. 
6. Beyersmann $D$, Hartwig A. Carcinogenic metal compounds: recent insight into molecular and cellular mechanisms. Arch Toxicol 2008; 82(8): 493e512.

7. Eybl V, Kotyzova D, Koutensky J. Comparative study of natural antioxidants - curcumin, resveratrol and melatonin - in cadmium-induced oxidative damage in mice. Toxicology 2006; 225 (2-3):150-156.

8. de la Torre R, Covas MI, Pujadas MA, Fito M, Farre M. Is dopamine behind the health benefits of red wine? Eur J Nutr 2006; 45 (5):307-310.

9. Yang $X$, Jing $T, L i Y$, He $Y$, Zhang $W$, Wang $B$, Xiao $Y$, Wang $W$, Zhang $J$ et al. Hydroxytyrosol Attenuates LPSInduced Acute Lung Injury in Mice by Regulating Autophagy and Sirtuin Expression. Curr Mol Med 2017; 17 (2) :149-159.

10. Cao K, Xu J, Zou X, Li Y, Chen C, Zheng A, Li H, Li H, Szeto $I M$, Shi $Y$ et al. Hydroxytyrosol prevents dietinduced metabolic syndrome and attenuates mitochondrial abnormalities in obese mice. Free Radic Biol Med 2014; 67: 396-407.

11. Merra E, Calzaretti G, Bobba A, Storelli MM, Casalino E. Antioxidant role of hydroxytyrosol on oxidative stress in cadmium-intoxicated rats: different effect in spleen and testes. Drug Chem Toxicol 2014; 37 (4):420-426.

12. Pei YH, Chen J, Xie L, Cai XM, Yang RH, Wang, Gong J. Hydroxytyrosol Protects against Myocardial Ischemia/Reperfusion Injury through a PIJK/AktDependent Mechanism. Mediators Inflamm 2016; 1232103.

13. Ohkawa H, Ohishi N, Yagi K. Assay for lipid peroxides in animal tissues by thiobarbituric acid reaction. Anal Biochem 1979; 95 (2) :351-358.

14. Levine RL, Garland D, Oliver CN, Amici A, Climent I, Lenz AG, Ahn BW, Shaltiel S, Stadtman ER. Determination of carbonyl content in oxidatively modified proteins. Methods Enzymol 1990; 186:464-478.

15. Boujelben M, Gorbel F, Vincent C, Macni-Ayadi F, Guermazi F, Croute F, El-Feki A. Lipid peroxidation and HSP72/73 expression in rat following cadmium chloride administration: Interactions of magnesium supplementation. Exp Toxicol Pathol 2006; 57 (5-6): 437-443

16. Ali $F$, Naqvi SA, Bismillah M, Wajid N. Comparative analysis of biochemical parameters in diabetic and non- diabetic acute myocardial infarction patients. Indian Heart J 2016; 68 (3):325-331.

17. Granados-Principal S, El-Azem N, Pamplona R, RamirezTortosa C, Pulido-Moran M, Vera-Ramirez, Quiles JL, Sanchez-Rovira $P$, Naudí $A$, Portero-Otin $M$. Hydroxytyrosol ameliorates oxidative stress and mitochondrial dysfunction in doxorubicin-induced cardiotoxicity in rats with breast cancer. Biochem Pharmacol 2014; 90 (1):25-33

18. Ninkov M, Popov Aleksandrov A, Demenesku J, Mirkov I, Mileusnic D, Petrovic A, Grigorov I, Zolotarevski L, Tolinacki M, Kataranovski D. Toxicity of oral cadmium intake: impact on gut immunity. Toxicol Lett 2015; 237 (2): 89-99

19. Fu P, Hu Q. 3, 4-Dihydroxyphenylethanol alleviates early brain injury by modulating oxidative stress and Akt and nuclear factor- $K B$ pathways in a rat model of subarachnoid hemorrhage. Exp Ther Med 2016; 11 (5): 1999-2004

20. Janabi M, Yamashita S, Hirano K, Sakai N, Hiraoka H, Matsumoto K, Zhang Z, Nozaki S, Matsuzawa Y. Oxidized LDL-induced NF-kappa $B$ activation and subsequent expression of proinflammatory genes are defective in monocyte-derived macrophages from $C D$ 36-deficient patients. Arterioscler Thromb Vasc Biol 2000; 20 (8): 1953-1960.

21. Zhong $Y W, W u$ J, Hu HL, Li W X, Zhong Y. Protective effect 3, 4-dihydroxyphenylethanol in subarachnoid hemorrhage provoked oxidative neuropathy. Exp Ther Med 2016; 12 (3):1908-1914.

22. Yuan $Y$, Wang $Y$, Hu FF, Jiang CY, Zhang YJ, Yang JL, Zhao SW, Gu JH, Liu XZ, Bian JC et al. Cadmium Activates Reactive Oxygen Species-dependent AKT/mTOR and Mitochondrial Apoptotic Pathways in Neuronal Cells. Biomed Environ Sci. 2016; 29 (2):117126.

23. Nazima B, Manoharan V, Miltonprabu S. Grape seed proanthocyanidins ameliorates cadmium-induced renal injury and oxidative stress in experimental rats through the up-regulation of nuclear related factor 2 and antioxidant responsive elements. Biochem Cell Biol 2015; 93 (2): 210-226.

24. Adams JM, Corey S. The Bcl-2 protein family: arbiters of cell survival. Science. 1998; 281(5381):1322-1326. 